\title{
Translation, cross-cultural adaptation and factor analysis of the Persian version of ankle instability instrument
}

\author{
Somayeh Mohamadi ${ }^{1}$, Ismail Ebrahimi*1 ${ }^{* 1}$ Mehdi Dadgoo ${ }^{1}$, Mahyar Salavati ${ }^{2}$, Ahmad Saeedi ${ }^{3}$, \\ Bijan Valiollahi ${ }^{4}$
}

Received: 28 Nov 2017

Published: 1 Sep 2018

\begin{abstract}
Background: Ankle Instability Instrument (AII) is a questionnaire for determination of ankle stability status. The aim of this study is to cross-culturally translate and investigate the reliability and validity of AII in a sample of Persian-speaking Iranians, suffering from ankle sprain.

Methods: One hundred twenty persons with a history of ankle sprain were recruited in the study. All participants completed the Persian version of Ankle Instability Instrument, Cumberland Ankle Instability Tool (CAIT), Foot and Ankle Ability Measure (FAAM) and Foot and Ankle Outcome Score (FAOS) at the baseline. Out of them, 60 randomly selected subjects completed the questionnaires once more, one week later. Face validity, Test-retest reliability, internal consistency, standard error of measurement, minimal metric detectable change, spearman's correlation coefficient and confirmatory factor analysis of AII measured. We used Lisrel v 8.80 software with significant level of $\mathrm{p}<0.05$.
\end{abstract}

Results: Persian version of AII is clear and unambiguous and its qualitative face validity was confirmed in the pilot study on the 20 subjects with a lateral ankle sprain. The interclass correlation coefficient, Cronbach's alpha, standard error of measurement and minimal metric detectable change were $0.93,0.87,0.81$ and 2.25 (95\% confidence interval, 0.85-0.96). The Spearman correlations coefficients between AII, and CAIT, FAAM and FAOS measures were $0.91,0.71$ and 0.69 respectively. The original three factor structure of AII was replicated based on the confirmatory factor analysis. Which showed an adequate fit of the model to the data and goodness-of-various fit indices.

Conclusion: The Ankle Instability Instrument Persian Version (AII-PV) is a reliable and valid measure for assessing the ankle stability status.

Keywords: Ankle sprain, Instability, Psychometric, Questionnaire, Persian, Confirmatory factor analysis

Copyright $₫$ Iran University of Medical Sciences

Cite this article as: Mohamadi S, Ebrahimi I, Dadgoo M, Salavati M, Saeedi A, Valiollahi B. Translation, cross-cultural adaptation and factor analysis of the Persian version of ankle instability instrument. Med J Islam Repub Iran. 2018 (1 Sep);32:79. https://doi.org/10.14196/mjiri.32.79

\section{Introduction}

Ankle sprain is one of the most common musculoskeletal injuries among physically active individuals (1). About 40 to 75 percent of people with a history of lateral ankle sprain may suffer from residual symptoms such as activity induced pain, recurrent swellings, giving way and recurrent injury (2). These residual symptoms may result in chronic ankle instability (CAI), both mechanical (MAI) and functional ankle instability (FAI) $(2,3)$. Studying individuals with MAI or FAI together as a single group (as CAI) may

\footnotetext{
Corresponding author: Dr Ismail Ebrahimi, ebrahimitakamjani.e@iums.ac.ir
}

1. Department of Physiotherapy, Iran University of Medical Sciences, Tehran, Iran. 2. Department of Physiotherapy, University of Social Welfare and Rehabilitation Sciences, Tehran, Iran.

3. Department of Statistical Research and Information Technology, Institute for Research and Planning in Higher Education, Tehran, Iran.

4. Department of Orthopedic Surgery, Iran University of Medical Sciences, Tehran, Iran. lead to inconsistent research results. Therefore, it is suggested to categorize, evaluate and treat these two condition $\mathrm{s}$ as separate clinical entities (3).

Approximately $40 \%$ of ankle sprains end to FAI which was first defined by Freeman in 1965 as a tendency for the foot to give way and has been considered as the primary criterion for the assessment and diagnosis of FAI (4). Thereafter, several definitions and inclusion criteria for FAI have been introduced, including the grade of the initial

$\uparrow$ What is "already known" in this topic:

Psychometric evaluation of Ankle Instability Instrument (AII) is a short and effective instrument for identifying Persian-speaking patients with functional ankle sprain and could be used for evaluating changes of ankle instability after treatment.

\section{$\rightarrow$ What this article adds:}

The newly developed Persian version of AII could be used as a reliable and valid tool for classifying patients with history of lateral ankle sprain and evaluate its recovery after rehabilitation. 
sprain, the presence and repetition of giving way, the frequency of ankle sprains and the last time when affected by ankle sprain $(5,6)$.

Because of the controversy about FAI characteristics, and the limited tests and screening instruments for the diagnosis of FAI, its primary definition is the frequent subjective report of ankle giving way (5). Ankle Instability Instrument (AII) was developed by Docherty in 2006 in an attempt to providing a more specific definition of FAI utilizing the common mentioned symptoms $(5,7)$. AII covers different aspects of ankle instability including the history, presence and severity of ankle instability and the state of functional performance. The acceptable reliability of AII have been reported by the developers (5).

AII has been frequently used to classify individuals with a history of ankle sprain, as well as to provide an inclusion criteria for identification of FAI (7-11). By means of AII, we can also determine changes in self-reported function after any intervention (7).

To the best of authors' knowledge, AII has not been translated and validated into Persian among Iranian subjects with a history of ankle sprain. The aim of the present study was to translate and cross-culturally adapt AII into Persian and to evaluate its psychometric properties in terms of reliability, validity and its factor structure in Persianspeaking patients with a lateral ankle sprain.

\section{Methods}

\section{Instruments}

AII is a discriminative instrument consisting of 9 Yes/No questions for evaluation of functional ankle instability (5). Its initial version included 21 questions about ankle instability. It was modified to a 9-item questionnaire without any subscales (5). From clinical point, AII is designed to classify participants as either having FAI or not at different time; after their injuries and treatments. The International Ankle Consortium, recommended the cut-off score of 5 YES response out of 99 Yes/No questions as the inclusion criteria for FAI (10).

To assess the convergent validation of AII, two other instruments, CAIT, FAAM and FAOS were used. Cumberland Ankle Instability Tool (CAIT) is another discriminative instrument, for measuring the severity of functional ankle instability. It is a 9-item questionnaire, with a maximum score of 30 and a cut-off point of 27.5, with lower scores indicating more severe functional ankle instabilities (9).

The Foot and Ankle Ability Measure (FAAM) is a region-specific questionnaire for measuring the function of foot and ankle. It is composed of two separately-scored dimensions; the Activity of Daily Living and Sports subscales with 21 and 8 items, respectively. Each item is scored on a 5 -point Likert scale from 0 to 4 representing different levels of difficulty in functional activities (unable to do, extreme difficulty, moderate difficulty, slight difficulty and no difficulty). Higher scores indicate higher levels of function for each subscale $(11,12)$.

The Foot and Ankle Outcome Score (FAOS) is a regionspecific evaluative questionnaire for measuring foot and ankle function. It is a 42 -item questionnaire with five subscales: Pain (9 items), Symptoms (7 items), Activity of
Daily Life (17 items), Sport and Recreation (5 items), and ankle and foot related Quality of Life (4 items). A fivepoint Likert scale ( 0 to 4 indicating severe, moderate, mild and no problem respectively) is used in FAOS to score each item, with higher scores indicating higher levels of function $(13,14)$.

\section{Participants}

A consecutive sample of 120 Persian-speaking people with a history of lateral ankle sprain were recruited from April 2016 to February 2017, through advertising in the university campus, hospitals, physiotherapy clinics and general community in Tehran, the capital of Iran. Participants were excluded if they had head, spine or lower extremity acute or chronic diseases or injuries within 3 months before participation; or any history of ankle fracture or surgery.

All participants signed an informed consent form approved by the Human Research Ethics Committee of the Iran University of Medical Sciences in Tehran, prior to participation. Ethic Committee Code number: IR.IUMS.REC. 1395.9211342211.

\section{Translation}

The International Quality of Life Assessment protocol was used to translate AII into Persian (14). The permission was obtained from the developer, Carrie L. Docherty, for translating the questionnaire.

Two native Persian-speaking translators translated the English version of AII into Persian. Then, in a meeting with the investigators, the translations were compared for inconsistencies in order to prepare a common preliminary Persian translation.

A native Persian expert translator evaluated the quality of the preliminary Persian questionnaire considering clarity, common language use, and conceptual equivalence and modified it if necessary. The final Persian version was backward translated into English by a native English-Persian speaking translator. The backward translation was checked and approved by the developer (Docherty) for conceptual equivalence. Later, its face validity was examined on 20 subjects with a history of ankle sprain in order to find any difficult, ambiguous and confusing items.

\section{Procedures}

At the first visit, all participants received four region-specific questionnaires (AII, CAIT, FAAM, and FAOS), in a random order. The questionnaires were filled for a second time by 60 participants one week later to be used for testretest reliability. Participants with any change in their health status during the test-retest period were excluded from retest evaluation. During all test sessions, an investigator was present to ensure that no external distractions occurred during the testing period. Respondents were allowed to have as much time as necessary to complete the questionnaires. The scores of all questionnaires collected at the first session were used to evaluate floor and ceiling effects, construct validity and factor structure. 


\section{Statistical Analysis}

Floor and ceiling effects: Floor or ceiling effects evaluate the instrument's ability in discriminating subjects with different levels of the studied attribute (15). floor or ceiling effects are present if more than $15 \%$ of the sample has the minimum or maximum of AII score range, respectively (14).

Internal Consistency: The homogeneity of the AII items was evaluated by calculating internal consistency using Cronbach's alpha coefficients. Coefficients above 0.70 is considered as acceptable internal consistencies (16).

Test-Retest reliability: In order to examine relative reliability, two-way random effects model of Interclass Correlation Coefficients $\left(\mathrm{ICC}_{2,1}\right)$ and its $95 \%$ confidence intervals (95\% CIs) for questionnaire scores in test and retest sessions were calculated. ICCs above 0.70 is considered as good reliability levels (14). In addition, Standard Errors of Measurement (SEM) and Minimally Metric Detectable Changes (MMDC) were calculated to examine absolute test-retest reliability. SEM is the square root of the mean square error term from analysis of variance as an estimate of measurement precision associated with repeated measurements. Observed SEM values were used for computing the MMDC which was defined as $95 \%$ CI of SEM $( \pm 1.96 \times \sqrt{2} \times \mathrm{SEM})$. MMDC is a measure of the minimal change in a variable which can be considered reliably beyond measurement error $(15,17)$.

\section{Validity}

Construct validity: Convergent validity as a measure of the relationship between the scores of an intended instrument with other validated and conceptually similar/correlated instruments was assessed (9). Spearman's correlation coefficients between AII and CAIT, FAAM and FAOS were computed. Coefficients below 0.30 , between 0.30 and 0.60 , and above 0.60 were considered as weak, moderate and strong correlations, respectively (14).

Factorial validity: Confirmatory Factor Analysis (CFA) was conducted to confirm the factor structure of the AII original version. According to Docherty, AII consists of three factors, including Severity of Initial Ankle Sprain, History of Ankle Instability and Instability during Activities of Daily Life.

According to Bollen and Long, recommendations (18), evaluation of each model should be based on a variety of fit measures to test the goodness of fit. In the present study, model fit was assessed using a number of goodness-of-fit indices including $\chi^{2}$, Mean Square Error of Approximation (RMSEA), goodness-of-fit index (GFI), adjusted goodness-of-fit index (AGFI) and Comparative Fit Index (CFI) by Lisrel $v 8.80$ software and the level of significant was set at $\mathrm{p}<0.05$.

\section{Results}

\section{Translation}

The preliminary Persian version of AII as well as its backward translated version received no significant comments on the translation quality and conceptual equivalence. The respondents reported no difficulty in understanding and completing the Persian version of AII during the pilot study. Therefore, the preliminary Persian version of AII questionnaire was satisfactorily face valid.

\section{Descriptive Statistics}

The descriptive profile of the participants are shown in Table 1. All of the subjects had sprained ankles and were between 20-50 years of age. AII scores showed a mean (SD) of 5.77 (2.48), a median of 6 and a range between 1 and 9.

\section{Floor or ceiling effects}

Less than $15 \%$ of participants had the lowest or highest possible scores of the total score of AII. No floor or ceiling effects of the Persian AII observed (Table 2).

\section{Reliability}

The values of Cronbach's alpha coefficients, ICC, SEM and MMDC for AII total score and its factors are shown in

Table 1. Descriptive profile of the study participants $(n=120)$

\begin{tabular}{lccc}
\hline Variable & \multicolumn{1}{c}{ Mean (SD) } & $\mathrm{N}(\%)$ \\
\hline Age & & $33.79(7.35)$ & - \\
Height & & $80.71(16.15)$ & - \\
Weight & $170.5(10.65)$ & - \\
BMI & & $27.85(5.05)$ & - \\
Sex & Female & - & $59(49.2)$ \\
& Male & - & $61(50.8)$ \\
Affected Side & Right & - & $51(42.5)$ \\
& Left & - & $69(57.5)$ \\
\hline
\end{tabular}

Table 2. Floor and ceiling effects of total of Persian version AII $(\mathrm{n}=120)$

\begin{tabular}{|c|c|c|c|c|c|c|}
\hline Scale & Min & Number & Floor $(\%)^{*}$ & Max & Number & Ceiling $(\%)^{* *}$ \\
\hline
\end{tabular}

\begin{tabular}{lllllll}
\hline AII & 1 & 3 & 2.5 & 9 & 16 & 13.33
\end{tabular}

*The percent of participant who gained minimum score

**The percent of participant who gained maximum score

Table 3. Reliability measures for the Persian version of AII and its factors $(n=60)$

\begin{tabular}{lcccc}
\hline variable & ICC $(95 \% \mathrm{CI})$ & Cronbach's $\alpha$ & SEM & MMDC \\
\hline AII & $0.93(0.85-0.96)$ & 0.87 & 0.81 & 2.25 \\
Factor 1 & $0.75(0.61-0.85)$ & 0.72 & 2.52 & 6.98 \\
Factor 2 & $0.79(0.67-0.87)$ & 0.78 & 2.48 & 6.87 \\
Factor 3 & $0.83(0.73-0.9)$ & 0.9 & 1.1 & 3.05 \\
\hline
\end{tabular}




\begin{tabular}{llc} 
Table 4. Reliability and Item-Scale analysis of the AII (n=60) & \multicolumn{1}{c}{$\begin{array}{c}\text { Adjusted item-scale cor- } \\
\text { relation }\end{array}$} \\
\hline Subscale & \multicolumn{1}{c}{$\begin{array}{c}\text { Cronbach alpha if the item is } \\
\text { deleted }\end{array}$} & 0.62 \\
\hline Severity of initial ankle & 2) Consultation with a physician & 0.19 \\
sprain & 2a) Severity of ankle sprain & 0.81 \\
& 3) Need for weight bearing assistant & 0.34 \\
& 3a) length of time weight bearing assistant was & 0.75 \\
needed & 0.25 \\
History of ankle instability & 0.87 \\
& 1) History of sprained ankle & 0.31 \\
& 4) History of ankle giving way & 0.79 \\
4nstability during activity & 6) unstable on uneven ground & 0.65 \\
of daily life & 7) unstable during recreational or sport activities & 0.72 \\
& 5) unstable on a flat surface & 0.74 \\
\hline
\end{tabular}

Table 3 .

Item-Scale analysis of the AII in (Table 4) shows the questions $2 \mathrm{a}, 3 \mathrm{a}$ and $4 \mathrm{a}$ don't have high reliability coefficients (the inter-item reliability coefficient under 0.5 ) and they could be considered for deletion/revision in future studies in Persian version to create an acceptable Cronbach's alpha value and increase reliability.

\section{Construct Validity}

Convergent validity was studied by evaluating the correlations between the AII and other theoretically-related questionnaires. The scores of AII and CAIT, which are expected to measure similar constructs, showed a high significant positive spearman correlation coefficient $(\mathrm{p}<0.001$, $\mathrm{r}=0.91$ ), the other correlations were positive and moderate ( 0.91 and 0.69 for FAAM and FAOS respectively).

CFA was performed on the covariance matrix of AII items. The three-factor model, originally proposed by Docherty et al. (5) specifies a pattern of correlations between the factors and items. The hypothesized three-factor model, fitted the data well according to the values of chi-square, RMSA, GFI, AGFI and CFI which were $334.9(\mathrm{p}=0.12)$, $0.08,0.77,0.85,0.76$ respectively. The fitting model and its parameter estimates are shown in Fig. 1. The factor loadings ranged between 0.44 and 0.76 . The correlations between the three factors are depicted as curved arrows between the circles which ranged between 0.25 and 0.51 .

Consistent with its original version of AII and other ankle questionnaires, our results showed that the Persian version of AII, is a reliable and valid measure of ankle instability status.

\section{Discussion}

The original English version of this instrument showed high test-retest reliability and internal consistency (ICC= 0.95 , Cronbach's $\alpha=0.89$ ) which is comparable to the result of the present study (ICC $=0.93$, Cronbach's $\alpha=0.87$ ) (5).

Hadadi et al. on 105 subjects with a history of ankle sprain and 30 control subjects, reported ICCs of CAIT questionnaire, 0.95 and 0.91 for right and left ankles, respectively. Moreover, Cronbach's alpha coefficients were higher than 0.70 for both ankles and in both groups (20).

The psychometric properties of Persian AII were in agreement with other Persian versions of region-specific questionnaires including FAAM and FAOS. Mazaheri et al
(17) found that Cronbach's alpha coefficient of 0.97, ICC of 0.98 and SEM of 3.13 for the ADL subscale of the Persian version of FAAM. They also reported a Cronbach's alpha coefficient of 0.94 , ICC of 0.98 and SEM of 3.53 for Sports subscales of the Persian version of FAAM.

Negahban et al (14) concluded that all of the Persian FAOS subscales have very high ICCs, ranging from 0.92 to 0.96 , with most of the subscales' Cronbach's alpha coefficients above 0.7 . Therefore, the Persian version of AII has a test-retest reliability comparable to other ankle instruments, indicating its effectiveness in both the clinical and research settings.

The use of AII to identify the presence of functional ankle instability is suggested (21). On the first and second trials

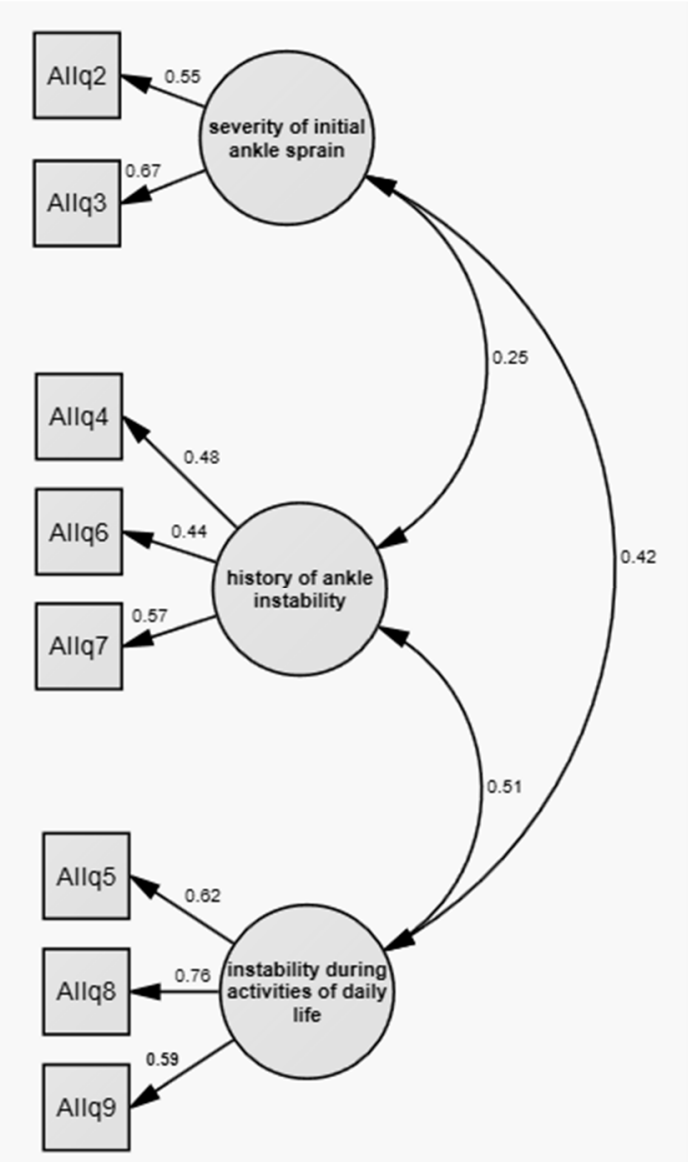

Fig. 1. Standardized factor loadings_of the 3-factor model of Docherty in patients with a sprained ankle. 
of measurement, 45 and 46 participants were identified as having FAI, respectively, 45 subjects common between these two trials. One subject without FAI on the first trial showed one score increase on her second trial. This slight disparity might be due to the borderline score on the first trial. In general, AII was shown to be an instrument by which subjects with sprained ankles could report their symptoms on successive occasions with a high level of reliability and can, therefore, be used in order to discriminate participants with and without FAI.

In addition, significant associations were found between AII and CAIT, FAAM, and FAOS. The correlations were high for CAIT to moderate for other scales. As AII is developed as a measure to identify FAI, stronger correlation between AII and CAIT as another discriminative questionnaire was not surprising. This is consistent with the previous studies regarding the relationships among different clinical and functional constructs in people with a history of ankle sprain $(17,21)$. As an instance, Hadadi et al in 105 participants with a history of ankle sprain found a significant correlation between FAAM and pain intensity $(\mathrm{r}=0.64)$ (21). Additionally, Mazaheri et al reported moderate correlations between Persian FAAM and SF-36 subscales (17).

The moderate correlations of Identification of Functional Ankle Instability (IdFAI) with FAAM and FAOS scores may be explained by the diference of constructs which these scales are designed to measure. Although FAAM and FAOS are foot- and ankle-specific instruments, they are specifically developed to measure a variety of clinical and functional outcomes in people with foot and ankle injuries. A wide variety of factors contribute to the clinical manifestations and functional disability in people with an ankle sprain. Therefore, it seems reasonable that these outcomes are only partly determined by, and consequently correlated with, the degree of ankle instability $(14,17)$.

CFA on AII scores of subjects with a history of lateral ankle sprain showed that a three-factor model of the AII proposed by Docherty et al (5) fitted well to our data according to the reference data points. All the calculated indices were between zero and one with high values which indicate a good fit of three-factor model (19). The Docherty's model also provided estimates of the factor loadings and correlations between the AII factors. All factor loadings were significant so items were interpreted as valid parts of the relevant factors. As the results showed the satisfactory fit for a three-factor model, we recommend using this version of the AII and its 3 factors in both clinical practice and research setting.

\section{Conclusion}

The Persian version of AII may be considered as a simple, reliable, and valid questionnaire for identifying the severity of functional ankle instability, measuring treatment outcome, and monitoring progress. Using AII to diagnose and classify subjects with FAI, will enable researcher and clinicians more homogenous subject groups to be determined and compared in the future studies because this instrument is useful to identify the degree of ankle instability.

\section{Limitations}

Therefore, the results do not necessarily indicate the ability of the questionnaire to show the effectiveness of different interventions. Subjects with severe (high grades) ankle sprain were dominant in our sample, which limits the generalizability of the results to the patients with mild and moderate sprains.

\section{Conflict of Interests}

The authors declare that they have no competing interests.

\section{References}

1. Doherty C, Delahunt E, Caulfield B, Hertel J, Ryan J, Bleakley C. The Incidence and Prevalence of Ankle Sprain Injury: A Systematic Review and Meta-Analysis of Prospective Epidemiological Studies Sports Medicine. Sports Med. 2014;44(1):123-40.

2. Wikstrom EA, Tillman MD, Borsa PA. Detection of Dynamic Stability Deficits in Subjects with Functional Ankle Instability. Med Sci Sports Exerc. 2005;37(2):169-75.

3. Brown CN, Padua DA, Marshall SW, Guskiewicz KM. Variability of motion in individuals with mechanical or functional ankle instability during a stop jump maneuver. Clin Biomech. 2009;24(9):762-8.

4. Freeman MA, dean MR, hanham IW. The etiology and prevention of functional instability of the foot. the journal of bone and joint surgery. 1965;47(4):678-85.

5. Docherty CL, Gansneder BM, Arnold BL, Hurwitz SA. Development and Reliability of Ankle Instability Instrument. $J$ Athl Train. 2006;41(2):154-8

6. Ross SE, Guskiewicz KM, Gross MT, YuB. Assessment Tools for Identifying Functional Limitations Associated With Functional Ankle Instability. Journal of Athletic Training. 2008;43(1):44-50.

7. Houston MN, Hoch JM, Hoch MC. Patient-Reported Outcome Measures in Individuals With Chronic Ankle Instability: A Systematic Review. J Athl Train. 2015;50(10):1019-33.

8. Delahaunt E, Coughlan G, Caulfield B, al. e. Inclusion Criteria When Investigating Insufficiencies in Chronic Ankle Instability. Med Sci Sports Exerc. 2010;42(11):2106-21.

9. Hiller CE, Refshauge KM, Bundy AC, Herbert RD, Kilbreath SL. The Cumberland Ankle Instability Tool: A Report of Validity and Reliability Testing. Arch Phys Med Rehabil. 2006;87(9):1235-41.

10. Gribble PA, Bleakley CM, Caulfield BM, Caulfield B, Docherty $\mathrm{CL}$, Fourchet F, et al. Selection criteria for patients with chronic ankle instability in controlled research: a position statement of the international ankle consortium. J Orthop Sports Phys Ther. 2013;43(8):585-91.

11. Kivlan BR, Martin RL, Wukich DK. Responsiveness of the foot and ankle ability measure (FAAM) in individuals with diabetes. Foot. 2011;21(2):84-7.

12. Measure in Athletes With Chronic Ankle Instability. Journal of Athletic Training. 2008;43(2):179-83.

13. Göksel Karatepe A, GünaydÂn R, Kaya T, KarlÂbao U, Özbek G. Validation of the Turkish version of the foot and ankle outcome score. Rheumatol Int. 2009; 30 (2):169-173.

14. Negahban H, Mazaheri M, Salavati M, al. e. Reliability and validity of the Foot and Ankle Outcome Score: a validation study from Iran. Clin Rheumatol. 2010;29(5):479-86.

15. Shanbehzadeh S, Salavati M, Tavahomi M, Khatibi A, Talebian S, Khademi-Kalantari K. Reliability and Validity of the Pain Anxiety Symptom Scale in Persian Speaking Chronic Low Back Pain Patients. Spine. 2017.

16. Gomez-Perez L, Lopez-Martınez AE, Ruiz-Parraga GT. Psychometric Properties of the Spanish Version of the Tampa Scale for Kinesiophobia (TSK). J Pain. 2011;12(4):425-35.

17. Mazaheri M, Salavati M, Negahban H, al. e. Reliability and validity of the Persian version of Foot and Ankle Ability Measure (FAAM) to measure functional limitations in patients with foot and ankle disorders. Osteoarthr Cartil. 2010;18(6):755-9.

18. Bollen KA, Long JS. Introduction. In: Bollen KA, Long JS, eds. Testing Structural Equation Models. Newbury Park, CA: Sage; $1993 ; 1-9$ 
19. Goubert L, Crombez G, Van Damme S, al. e. Confirmatory Factor Analysis of the Tampa Scale for Kinesiophobia Invariant Two-Factor Model Across Low Back Pain Patients and Fibromyalgia Patients. Clin J Pain. 2004;20(2):103-10.

20. Hadadi M, EbrahimiTakamjani I, Mosavi ME, al. e. Cross-cultural adaptation, reliability, and validity of the Persian version of the Cumberland Ankle Instability Tool. Disabil Rehabil. 2017;39(16):1644-9.

21. Fox J, Docherty C, Schrader J, Applegate T. Eccentric Plantar-Flexor Torque Deficits in Participants with Functional Ankle Instability. J Athl Train. 2008;43(1):51-4. 